\title{
Development of Smart Cities with Fog Computing and Internet of Things
}

\author{
Kong-Long Lai, \\ Department of Electrical Engineering, \\ Da-Yeh University, Taiwan. \\ Dr. Joy Iong Zong Chen, \\ Professor, Department of Electrical Engineering, \\ Da-Yeh University, Taiwan. \\ Email id: jchen@mail.dyu.edu.tw
}

\begin{abstract}
In construction of smart cities, Internet of Things and Fog computing have a crucial role to play which requires the need for management and exchange of large amount of information. Both Internet of Things as well as Fog computing are two predominant fields that have emerged in recent years to enable the development of transportation, tourism, industries as well as business in a proficient manner. Hence the introduction of a smart city will require proper study as well as ways to improve the strength's of the city using technological advancement. This will also enhance the strength of city in many fronts. In this paper, we have examined the positive aspects of fog computing using an IoT architecture that is integrated with fog computing in order to address the issues of network scalability and big data processing. Accordingly, the architecture of the IoT system is built such that the smart city will be able to function in a more efficient manner by means of network transmission, information processing and intelligent perceptions.
\end{abstract}

Keywords: Internet of Things; Smart City; Fog Computing; Cloud Computing; Information gathering

\section{Introduction}

As science and technology progresses in a quick manner, there have been new developments in the area of cloud computing and internet of things along with its applications. This will improve the way in which the city operates and will attain the goal of urban intelligent 
Journal of Ubiquitous Computing and Communication Technologies (UCCT) (2021)

Vol.03/ No.01

Pages: 52-60

https://www.irojournals.com/jucct/

DOI: https://doi.org/10.36548/jucct.2021.1.006

construction. As the urban areas begin developing, they have progressed quickly towards becoming smart cities [1]. Hence focus on the technology used for Internet of Things and Cloud Computing has become a mandatory to promote the realization of smart cities. On analyzing and examining the various theories on Smart cities, Internet of Things and cloud computing, we have proposed the use of IoT and Cloud computing to build smart cities, which will eventually enable the rapid development of smart cities. Using fog computing, it is possible to use to full capacity the control, communication, storage and distributed computing capabilities of the network environment. Using collaborative service architecture and resource sharing mechanism, it is possible to attain better accuracy in control, deciding and analysis, quicker response, secure decentralized service architecture, lower data transmission load, sturdier localized computing capacity and quicker service response time [2]. As the popularization, maturity and continuous development of fog computing technology improves, there will be a subsequent increase in the efficiency and convenience of using the Internet of Things. Both cloud computing as well as fog computing complement each other, giving everyone the right to intelligent services and enabling all cities to utilize the development power through interconnected technological advancements.

\section{Related Works}

In [3] the Internet of Things incorporated in the development of smart cities overseas has been studied along with the use of micro-smart grids to further equip the smart cities to grow. It was believed that to manage the already existing resources, the use of communication technologies, new information and smart cities in the future is crucial. Similarly, in [4], using large scale wireless sensor networks to operate a street lamp remote control will also decrement the maintenance and power costs. Researchers in [5] studied the urban traffic prediction of ITS using information obtained real-time and also put forth various modelling methods to predict urban traffic. In [6], the use of big data and its feasibility were discussed when they were used in smart city construction. It was believed that with the help of big data, it is possible to give valuable measures for cities using the large database of data received from several data sources to help in the process of taking decisions. There is also record of work in domestic level where heterogeneous integration of resources like big data [7] in order to build a simple architecture of resource database and establishing a combination of 
Journal of Ubiquitous Computing and Communication Technologies (UCCT) (2021)

Vol.03/ No.01

Pages: 52-60

https://www.irojournals.com/jucct/

DOI: https://doi.org/10.36548/jucct.2021.1.006

structured and unstructured data in the retrieval framework to meet the requirement of smart cities. Researchers also examined the different parts of the smart city and the security risk involved in harming the information stored [8]. Accordingly, several guarantee systems were formulated for the smart city based on disaster preparedness, emergency, technology, management, system and organization.

According to the information utilization and data elements, some scholars in [9] observed how it was possible to use the data in constructing smart parks and cities and how to use the data open, under the criteria that the data mining technology is used according to the suggestions. Zigbee technology was also used as wireless network solutions for smart parks and smart homes. In the current scenario, there are some drawbacks faced by the government in building smart cities with maximum potential, such as poor degree of intelligence, high degree of design limitation, fragile security and high degree of data fragmentation. Using technological advancements such as big data, cloud computing and internet of things, it is possible to address these issues based on how it uses high coverage of network transmission technology and perceptual data technology. However, information on how to centralize polymorphic data and intelligently process unstructured data is difficult to achieve intelligent decision making and high data sharing process [10]. Hence, to address these issues, this paper integrates Internet of Things with fog computing to enable better development of smart cities. The basic aspect of using fog computing is to use virtual machines on the edge network of cloud processing servers. The work of data layer and control layer is completed with the help of wireless network access, making it possible to be incorporated on the local network itself. Thus it will also decrease the resource waste and computational burden [11]. Moreover, since fog nodes are located near the Internet of things, it will also decrease the delay occurring when transmitting data between the source and the destination. Internet of Things and application of cloud computing along with other methodologies will give the essential aid to efficiently develop urban construction [12].

\section{Proposed Work}

To enable seamless coverage of users, many routing devices or base stations in mobile networks have been introduced. Service to Internet of Things [13] is also possible with the 
Journal of Ubiquitous Computing and Communication Technologies (UCCT) (2021)

Vol.03/ No.01

Pages: 52-60

https://www.irojournals.com/jucct/

DOI: https://doi.org/10.36548/jucct.2021.1.006

help of these devices. Because of the vast characteristics of Internet of Things devices, which will help to enable various essentials for transmission, every routing equipment or base station must hold a collection of access modes like LTE, ZigBee, Bluetooth and Wi-Fi. This methodology of routing devices or base stations with many access means is known as the wireless gateway that is used to gather information or data. Due to the many advantages of Fog computing, it can be incorporated in the architecture of IoT. This will improve the effective means with which fog nodes can be used with these routing devices or base stations in order to process the data that is gathered. The proposed architecture of IoT using fog computing is represented in Fig.1. Here the fog nodes are connected to routing devices or base stations by means of fibers of high capacity to decrease the transmission delay that occurs while transferring data. Moreover, the fog nodes are located at the edge of cell network such that the same fog node can be used by different routing devices and base stations in order to process data. The previously existing architectures had poor scalability and low data processing capability. However, when processing with the aid of Fog node core network, it is possible to overcome these drawbacks.

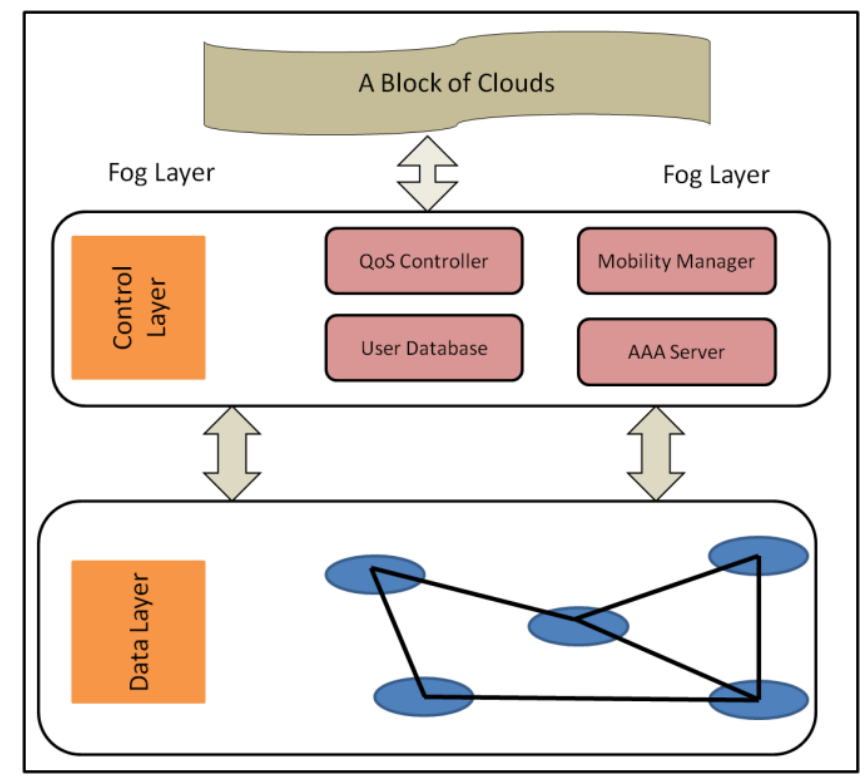

Fig.1. Architecture of the Proposed Methodology

Consider an intelligent video surveillance system that uses the methodology of detecting the target, analyzing its behaviour and judging the behaviour. Modelling method based on 
Journal of Ubiquitous Computing and Communication Technologies (UCCT) (2021)

Vol.03/ No.01

Pages: 52-60

https://www.irojournals.com/jucct/

DOI: https://doi.org/10.36548/jucct.2021.1.006

background detection is used for target entry scene. The constraint equation is computed for the detected image. Presence of anomaly is determined using convolutional neural network and accordingly, the user is alerted. The constraint equation is expressed in equation (1)

$$
I\left(a_{x}, b_{x}, x\right)=I\left(a+\Delta a_{x}, b+\Delta b_{x}, x+\Delta x\right)
$$

Where $x$ and $\Delta x$ represents two frames of pixel movement. When this movement is very small, the equation can be further reduced to:

$$
I\left(a+\Delta a_{x}, b+\Delta b_{x}, x+\Delta x\right)=I\left(a_{x}, b_{x}, x\right)+\frac{\partial \mathrm{F}}{\partial \mathrm{a}} \Delta a+\frac{\partial \mathrm{F}}{\partial \mathrm{b}} \Delta b+\frac{\partial \mathrm{F}}{\partial \mathrm{a}} \Delta x
$$

Using simplifying formula, the relation can be further reduced to:

$$
\frac{\partial \mathrm{F}}{\partial \mathrm{a}} \frac{\Delta a}{\Delta x}+\frac{\partial \mathrm{F}}{\partial \mathrm{b}} \frac{\Delta b}{\Delta x}+\frac{\partial \mathrm{F}}{\partial \mathrm{t}} \frac{\Delta t}{\Delta x}
$$

Here $a$ and $b$ will represent the direction of $I(a, b, t)$ such that at those points, the direction difference is represented as $\frac{\partial F}{\partial a}, \frac{\partial F}{\partial b}, \frac{\partial F}{\partial t}$. In equation, the composition of $a$ and $b$ will be indicative of the optical flow direction of $I(a, b, t)$ such that:

$$
I_{a} Z_{a}+I_{b} Z_{b}=-I_{x}
$$

This equation in the direction of $\mathrm{a}$ and $\mathrm{b}$ in the optical vector of $Z_{a}, Z_{b}$ where $I_{a}, I_{b}$ and $I_{x}$ which gives the deviation in the direction of corresponding of image ( $a, b, x)$. Hence there are 5 unknowns such that the equation can be represented for a $3 \times 3$ window.

$$
\left\{\begin{array}{c}
I_{a 1} Z_{a}+I_{b 1} Z_{b}=-I_{x 1} \\
I_{a 2} Z_{a}+I_{b 2} Z_{b}=-I_{x 2} \\
\cdot \\
\cdot \\
\cdot \\
I_{a 9} Z_{a}+I_{b 9} Z_{b}=-I_{x 9}
\end{array}\right.
$$


Journal of Ubiquitous Computing and Communication Technologies (UCCT) (2021)

Vol.03/ No.01

Pages: 52-60

https://www.irojournals.com/jucct/

DOI: https://doi.org/10.36548/jucct.2021.1.006

The proxy virtual processors are used in proxy virtual and dynamic processors are incorporated in the fog nodes located near the devices of IoT.
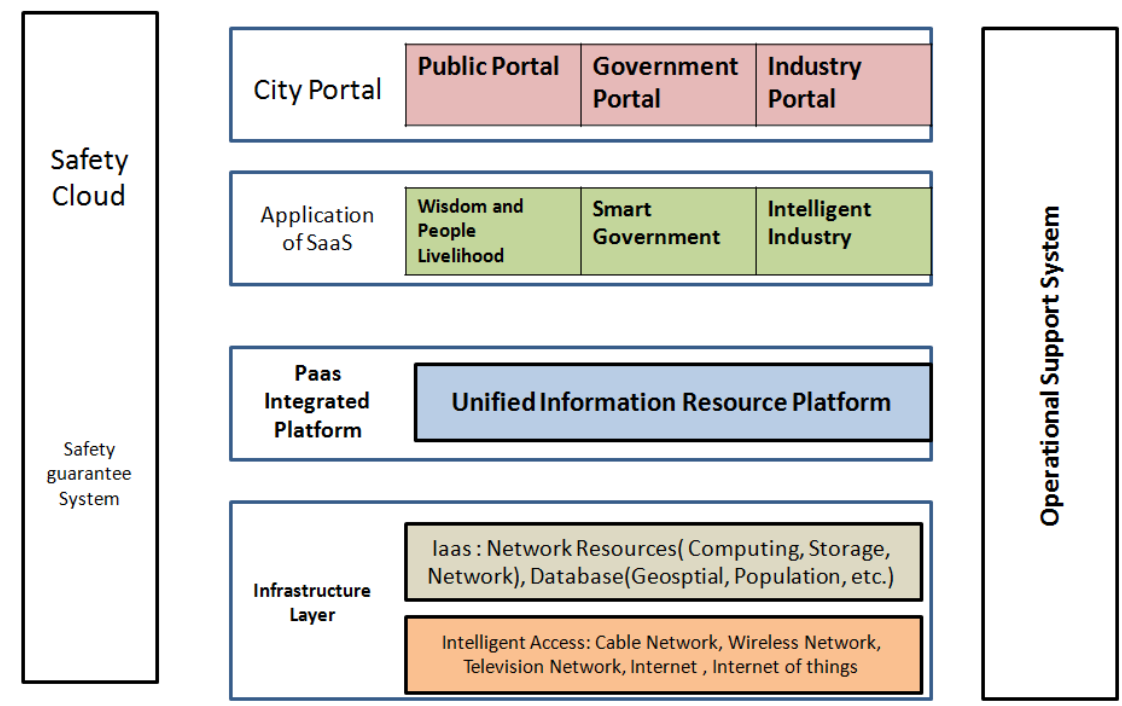

Fig. 2. Smart City Development Plan

A typical smart city development plan is laid out in Fig.2. In general, there are two schemes that can be used in virtual processors namely remote application and local application.

- The remote application virtual processors are used to process and transmit the data using the cloud nodes with the aid of various fog nodes. A good example is the use of traffic information in the proposed work which will give details on the road congestion information and accordingly, the optimal operation path can be provided to the user.

- The local application virtual processors are used to analyze the data that is obtained through the virtual servers. An example is the use of vehicle information to identify parking applications and to help the vehicle drivers to identify a suitable parking spot.

\section{Results and Discussion}

We experimented with the resource allocation time based the new service devices, idle service devices and various tasks involved. Here new services indicated the devices that are included within the time window to the device queue. We have considered a stable speed for 
Journal of Ubiquitous Computing and Communication Technologies (UCCT) (2021)

Vol.03/ No.01

Pages: 52-60

https://www.irojournals.com/jucct/

DOI: https://doi.org/10.36548/jucct.2021.1.006

the equipment such that it is maintained according to the subtasks in every cycle and is related to the arrival speed of the equipment. Fig.3.a and b show the allocation time for 100 subsets with respect to initial number of devices as 20 and 40. Similarly, Fig.4.a and b show the allocation time for 200 subsets with respect to initial number of devices as 20 and 40 . It is observed that the rejection rate of the neuron tasks is fixed at $80 \%, 60 \%, 30 \%$ and $10 \%$.

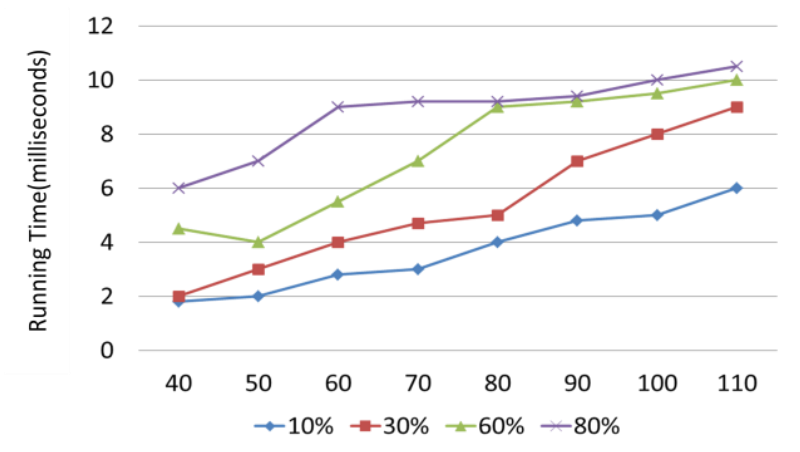

New equipment rate\%

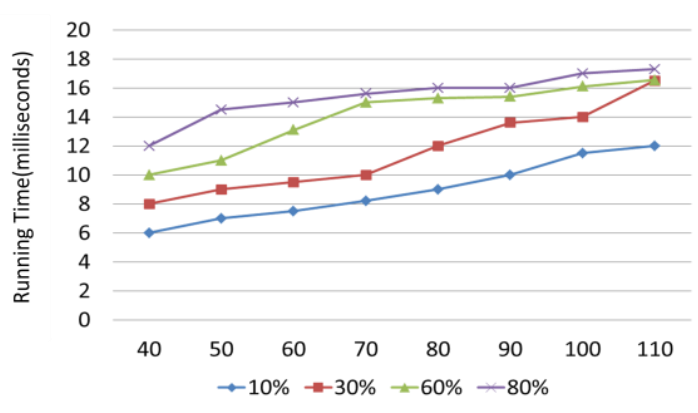

New equipment rate\%

Fig. 3. a. Allocation of time for 100 subtasks with 20 devices as initial number b. Allocation of time for 100 subtasks with 40 devices as initial number

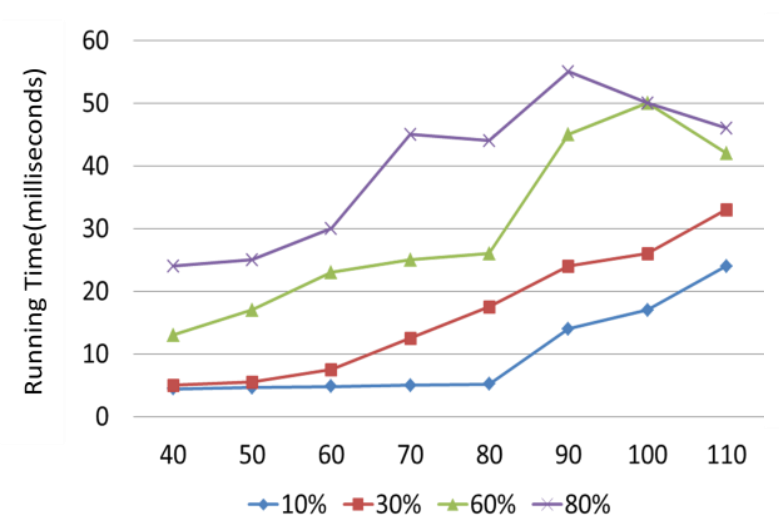

New equipment rate\%

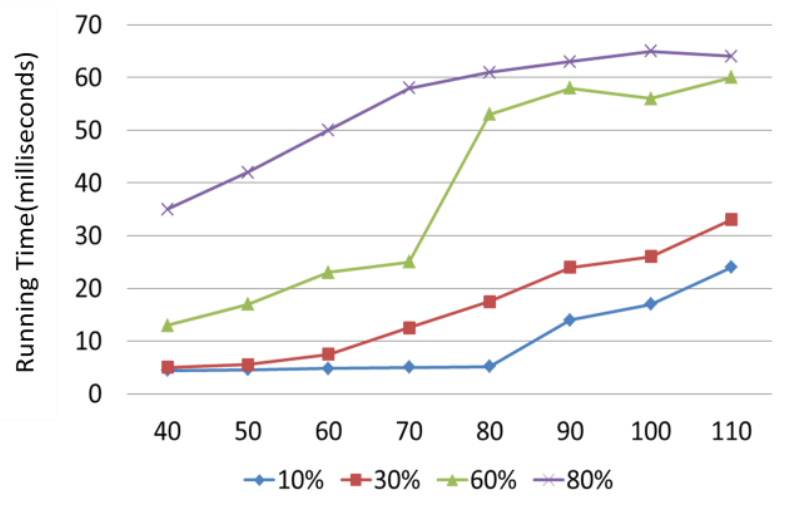

New equipment rate\%

Fig. 4. a. Allocation of time for 200 subtasks with 20 devices as initial number b. Allocation of time for 200 subtasks with 40 devices as initial number

\section{Conclusion}

The development and build of smart cities is a crucial part of the growth of the economy as well as the betterment of the people living in a country. However, in order to create a smart city, there is need for thinking about the rational ways to utilize the available urban resources such that it is possible to develop a long-term plan that operates without any hindrance 
Journal of Ubiquitous Computing and Communication Technologies (UCCT) (2021)

Vol.03/ No.01

Pages: 52-60

https://www.irojournals.com/jucct/

DOI: https://doi.org/10.36548/jucct.2021.1.006

towards completing it. Hence to satisfy the requirements, it is necessary to use Internet of Things and cloud computing on one platform. Advanced communication technology and information technology can be used in transportation, medical industry and other industries to meet the essentials of the development of those industries to ensure that the smart city construction takes place in a perfect manner. We have proposed a novel work that not only decreases the delay in task processing but will also reduce running time and task violation rate. Future research will be carried out on improving computing resource allocation based on storage capacity, communication capacity and computing power.

\section{References}

[1] Mahmud, R., Ramamohanarao, K., \& Buyya, R. (2020). Application management in fog computing environments: A taxonomy, review and future directions. ACM Computing Surveys (CSUR), 53(4), 1-43.

[2] Tange, K., De Donno, M., Fafoutis, X., \& Dragoni, N. (2020). A systematic survey of industrial internet of things security: Requirements and fog computing opportunities. IEEE Communications Surveys \& Tutorials, 22(4), 2489-2520.

[3] Wu, D., \& Ansari, N. (2020). A cooperative computing strategy for blockchain-secured fog computing. IEEE Internet of Things Journal, 7(7), 6603-6609.

[4] Qiu, M., Kung, S. Y., \& Gai, K. (2020). Intelligent security and optimization in Edge/Fog Computing.

[5] Haghi Kashani, M., Rahmani, A. M., \& Jafari Navimipour, N. (2020). Quality of service-aware approaches in fog computing. International Journal of Communication Systems, 33(8), e4340.

[6] Shirley, D. Ruth Anita. "Systematic diagnosis of power switches." In 2014 International Conference on Embedded Systems (ICES), pp. 32-34. IEEE, 2014.

[7] Tychalas, D., \& Karatza, H. (2020). A scheduling algorithm for a fog computing system with bag-of-tasks jobs: Simulation and performance evaluation. Simulation Modelling Practice and Theory, 98, 101982.

[8] Puliafito, C., Goncalves, D. M., Lopes, M. M., Martins, L. L., Madeira, E., Mingozzi, E., ... \& Bittencourt, L. F. (2020). MobFogSim: Simulation of mobility and migration for fog computing. Simulation Modelling Practice and Theory, 101, 102062. 
Journal of Ubiquitous Computing and Communication Technologies (UCCT) (2021)

Vol.03/ No.01

Pages: 52-60

https://www.irojournals.com/jucct/

DOI: https://doi.org/10.36548/jucct.2021.1.006

[9] Zhou, C., Fu, A., Yu, S., Yang, W., Wang, H., \& Zhang, Y. (2020). Privacy-preserving federated learning in fog computing. IEEE Internet of Things Journal, 7(11), 1078210793.

[10] Ali, B., Pasha, M. A., ul Islam, S., Song, H., \& Buyya, R. (2020). A VolunteerSupported Fog Computing Environment for Delay-Sensitive IoT Applications. IEEE Internet of Things Journal, 8(5), 3822-3830.

[11] Shakya, S., Pulchowk, L. N., \& Smys, S. (2020). Anomalies Detection in Fog Computing Architectures Using Deep Learning. Journal: Journal of Trends in Computer Science and Smart Technology March, 2020(1), 46-55.

[12] Chen, J. I. Z., \& Smys, S. (2020). Interoperability Improvement in Internet of Things Using Fog Assisted Semantic Frame Work. Journal of trends in Computer Science and Smart technology (TCSST), 2(01), 56-68.

[13] Mugunthan, S. R. (2020). Decision Tree Based Interference Recognition for Fog Enabled IOT Architecture. Journal of trends in Computer Science and Smart technology (TCSST), 2(01), 15-25. 